\title{
Behaviour of manganese impurity in $\beta-\mathrm{ZnP}_{2}$
}

\author{
M. Kakazej, A. Kudin, M. Pinkovs'ka *, V. Tartachnyk* \\ National Dragomanov Pedagogical University, 9 Pirogov street, Kyiv, Ukraine, \\ Ph..221-99-71, Fax: 044-2350300 \\ E-mail: kudin2001@mail.ru \\ *Scientific Center «Institute for Nuclear Research» NAS of Ukraine, \\ 47 pr. Nauky, Kyiv 03028, Ukraine \\ Tel.265-37-49, Fax: 044-265-44-63 \\ E-mail:plitov@kinr.kiev.ua
}

\begin{abstract}
Behavior of manganese impurity in black zinc diphosphide was investigated for the first time by the EPR method at room temperature. The nature of basic singularities of an EPR spectrum was determined. The defect structure evolution of irradiated and annealed $\mathrm{ZnP}_{2}$ was studied by the method of the paramagnetic probe $\left(\mathrm{Mn}^{2+}\right)$.
\end{abstract}

Keywords: singularity, paramagnetic center, spin - Hamiltonian, defect, intersticial, vacancy, tetrahedron.

Paper received 14.09.01; revised manuscript received 27.11.01; accepted for publication 12.12.01.

\section{Introduction}

Black zinc diphosphide - a direct-zone semiconductor with an energy gap $E_{g}=1.33 \mathrm{eV}$ at $300 \mathrm{~K}$ that has high photoconducitivity in near infra-red (IR) region $\left(\lambda_{\max }=\right.$ $=1.06 \mu \mathrm{m})[1],-$ is promissing material for designing fast IR detectors [2,3] and displays a number of unique biexciton $[4,5]$ and gyrotropic [6] effects.

On the other hand, these devices are sensitive to electron irradiation. The linear dose dependence of the resistivity in the wide fluency region up to $10^{18} \mathrm{~cm}^{-2}[7]$ makes it possible to use $\mathrm{ZnP}_{2}$ diodes as sensors of electron irradiation. High-energy particle irradiation promotes purposeful control of their properties. However, defect structure of $\beta$ $\mathrm{ZnP}_{2}$ is not clear so far and needs further study.

The possibility to use paramagnetic centers as indicators of a local electrical field for study of radiation defects in semiconductors is well known. In the given work the method of a paramagnetic probe (Mn) was applied for an evaluation of changes of a defect structure of $\beta-\mathrm{ZnP}_{2}$ crystals under irradiation and thermal annealing.

According to the theory of the self-consistent method of the electron structure calculation of iron group transi- tional elements in binary phosphide, the minimal level depth $E_{a}$ of these metals from the valence zone can be described [8] by the formula:

$E_{a} \cong 0.5 E_{g}(1-f)$,

where $f$ is the magnitude of an ion degree. For $\beta-\mathrm{ZnP}_{2}$, where $f=0.42$ [9]), $E_{a} \cong 0.40 \mathrm{eV}$. In order to compare this value with the position of impurity levels appearing in the forbidden gap during irradiation and annealing, optical absorption and Hall effect measurements were carried out.

Experimental results are presented and main features of $\mathrm{ZnP}_{2}$ defect structure are discussed.

\section{Experiment}

Black $\mathrm{ZnP}_{2}$ crystals grown from a vapor phase were studied. The doping was carried out during synthesis of the composition. Manganese was incorporated in the form of phosphide. In order to receive the homogeneous distribu- 


\section{Kakazej et al.: Behaviour of manganese impurity in $\beta-\mathrm{ZnP}_{2}$}

tion of impurities in the volume of the crystals, the method proposed in [10] was used. The impurity concentration was controlled by the method of mass-spectrometry of secondary ions, using LAS-600 [11]. Roentgen-structural analysis was carried out using roentgenmeter TUR-62.

Crystals were irradiated by high-energy electrons ( $E=14 \mathrm{MeV})$ up to the fluence $\Phi=10^{17} \mathrm{~cm}^{-2}$ at room temperature. The isochronous annealing with the period of 15 minutes was carried out in special vacuum ampoule using a method proposed in [12].

Optical characteristics of crystals were measured at $T=77 \mathrm{~K}$ using a spectrometer of the Pye Unicam corporation, and electrical - by a five-probe method using highly sensitive amplifiers [13].

EPR spectra in the X-range were studied in powder samples by a radiospectrometer RE 1301 at room temperature and calculated by the technique given in [14-17].

\section{Results and discussion}

As one can see from the experiment, optical absorption of samples doped by manganese displays the shift of the edge of a fundamental absorption and a lack of selective bands in a spectral region from 0.1 up to $1.4 \mathrm{eV}$, comparing with initial spectra (Fig. 1).

Hall effect measurements have shown the drop of the hole concentration by 4 orders of value when $\mathrm{Mn}$ is incorporated into $\beta$ - $\mathrm{ZnP}_{2}$ (Table 1$)$.

In doped crystals, as one can see in the Table 1, the activation energy of the dominating acceptor level does not vary, the magnitude of mobility increases a bit, but its temperature dependence has remained without change (Fig. 2). All this indicates that in zinc diphosphide as well as in crystals $\mathrm{A}^{3} \mathrm{~B}^{5}[8]$ impurities of transitional metals with unfilled $3 \mathrm{~d}$ - shell form deep local levels with the

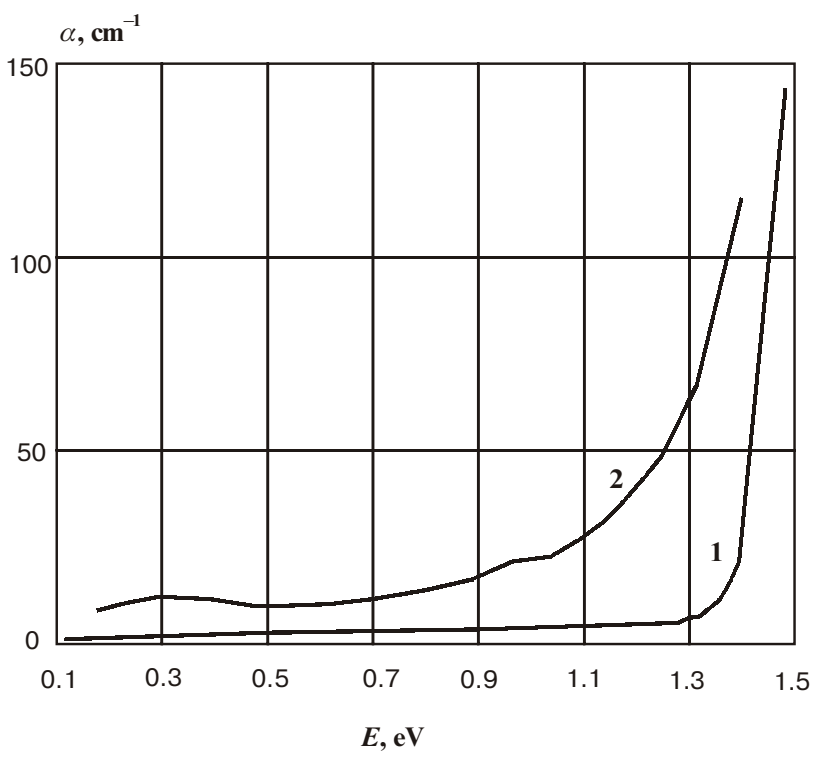

Fig. 1. Absorption spectra for initial (a) and manganese doped (b) black zinc diphosphide crystals
Table 1. Electrical parameters of black zinc diphosphide crystals

\begin{tabular}{llll}
\hline $\begin{array}{l}\text { \ Parameter } \\
\text { Crystal } \backslash\end{array}$ & $p, \mathrm{~m}^{-3}$ & $\begin{array}{l}\mu \cdot 10^{4}, \\
\mathrm{~m}^{2} / \mathrm{V} \cdot \mathrm{s}\end{array}$ & $E_{a}, \mathrm{eV}$ \\
\hline Initial & $6.8 \cdot 10^{14}$ & 48 & 0.36 \\
\hline $\mathrm{ZnP}_{2}: \mathrm{Mn}$ & $3.1 \cdot 10^{10}$ & 50 & 0.36 \\
\hline Anneal $T=800^{\circ} \mathrm{C}$ & $7 \cdot 10^{10}$ & 50 & 0.36 \\
\hline Anneal $T=850^{\circ} \mathrm{C}$ & $3 \cdot 10^{11}$ & 52 & 0.30 \\
\hline
\end{tabular}

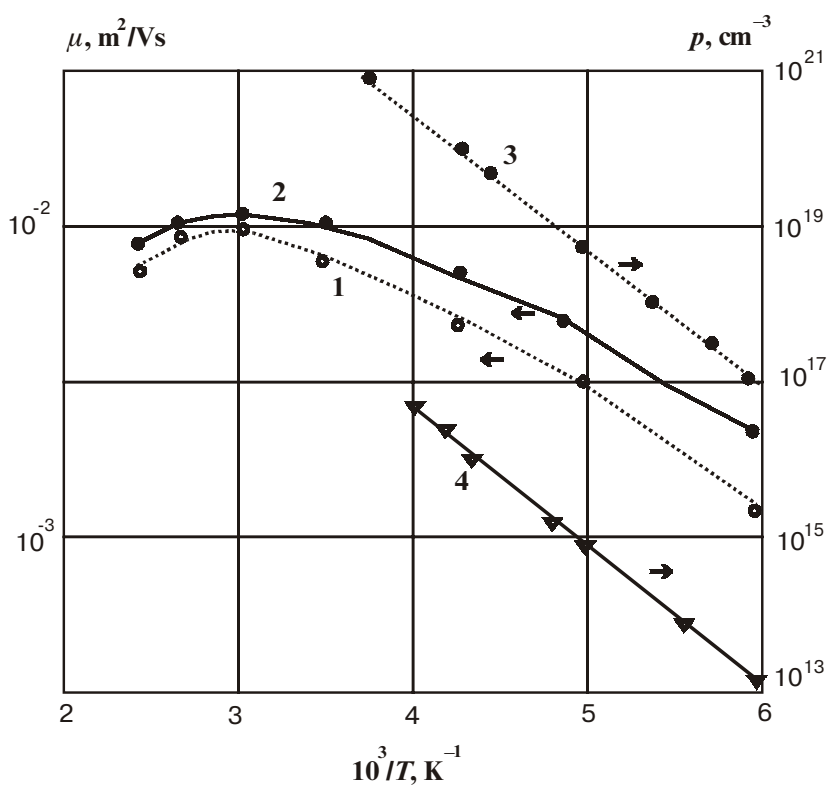

Fig. 2.Temperature dependences of the mobility and carrier concentrations for initial (doted lines) and manganese doped (solid lines) black zinc diphosphide crystals

small capture cross section of charge carriers in a forbidden zone.

As was established earlier in [18], initial crystals possess low thermal stability of main electrical parameters: at $T=760^{\circ} \mathrm{C}$ electroconductivity already increases by four orders, and varies ambiguously during farther heating. From the Table 1, it is clear that electrical parameters of manganese doped crystals are much more thermostable. Such property is characteristic for materials, in which doping impurities are in a substitution solution [19,20]. As it was shown in majority of works concerning EPR radiospectroscopy of wide-zone semiconductors [21-26], atoms of transitional metals can occupy interstitial positions (for example, in $\mathrm{SiC}$ [21]) or substitute metal (for example, in GaN [24]). For the definition of a manganese position in a lattice of $\beta$ $\mathrm{ZnP}_{2}$ crystals the spectra of an electron paramagnetic resonance were studied. To the beginning of our researches, the EPR spectra in complex phosphides $\mathrm{A}^{2} \mathrm{~B}^{5}{ }_{2}$ have not been studied jet, except of triple germanium diphosphide $\mathrm{ZnGe}$ $P_{2}$ [27-29]. Our experiments are the first of this kind. 


\section{Kakazej et al.: Behaviour of manganese impurity in $\beta-\mathrm{ZnP}_{2}$}

According to the concentration of manganese in synthesized substance, spectra of two types were obtained.

The first type (Mn content did not exceed 0.01 weight $\%$ ). EPR spectrum consists of four groups of lines nearly the same intensity (Fig. 3). Every group possesses six equidistant lines (Fig. 3, curve $a$ ), which responds to central transitions (CT) of $\mathrm{Mn}^{2+}$ ions in zinc diphosphide : $|1 / 2, m<\leftrightarrow|-1 / 2, m>$. The complicated picture of weak lines was observed near this intensive CT, which makes it unable to observe hyperfine forbidden transitions (HFT): $|1 / 2, m<\leftrightarrow|-1 / 2, m+1>$. In this case, parameters of a fine structure $(D$ and $E$ ) have a broad distribution, contrary to parameters $g$ and $A$, presented in Table 2.

Table 2. EPR parameters of singularities of spectra of p-type doped crystals

\begin{tabular}{ll}
\hline$g_{\text {ef }}$ & $A, \mathrm{mT}$ \\
\hline 20 & 5.4 \\
\hline 2.55 & 4.4 \\
\hline 2.17 & 6.2 \\
\hline 1.94 & 6.2 \\
\hline \hline
\end{tabular}

One can see that the magnitude of the parameter lies within limits 4.4-6.2 $\mathrm{mT}$, which is characteristic of compounds with the significant part of covalent bonds [15]. It is necessary to notice that the behavior of spectra is explained within the framework of the common analysis of unordering structures $[14,27]$.

In the second type EPR spectrum (with the Mn content up to 0,1 weight of $\%$ ) compared with the spectrum of the first type, the intensity of singularities with $g_{e f}=20$ is increased, the singularities with $g_{e f}=4.3$ and the broad band of Lorentz form in the region 270-370 mT appear (Fig. 3, curve $b$ ).

EPR spectra of manganese ions in the field of 270-370 $m T$

As one can see in Fig. 3, the band of Lorentz form has not a hyperfine structure (HFS) or bands are strongly superimposed. In order to define its basic characteristics the method of simultaneous observation of EPR spectrum in two substances was used: the unknown crystal and measured stand$\operatorname{ard}(\mathrm{MgO})$. The resulting spectrum is shown in Fig. 4. Using data of the measured standard spectrum, calculations of the $g$-factor and the width of a researched band were made:

$g_{e f}=\left(B_{l} / B_{2}\right) g_{1}=(3200 / 3165) 1.9880=2.0099$

$\Delta B=(65 \mathrm{~mm}) \cdot 2.18(\mathrm{Oe} / \mathrm{mm})=141.7 \mathrm{Oe}=14 \mathrm{mT}$.

If the width of individual lines caused by relaxation processes exceeds a distance between adjacent lines of the FS

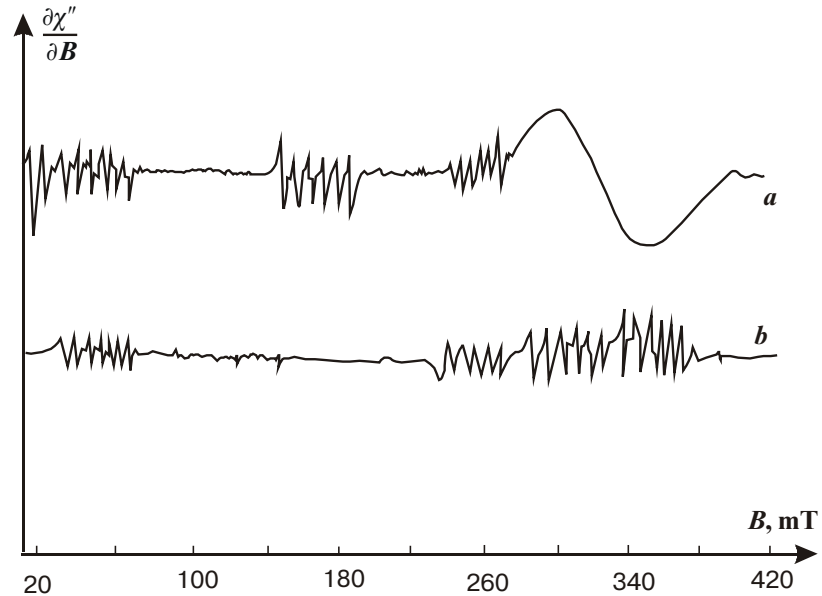

Fig. 3. EPR spectra for $\beta-\mathrm{ZnP}_{2}$ : Mn crystals with the different doping content: a) 0,01 weight $\%, b$ ) 0,1 weight $\%$.

and HFS, the structureless spectrum is observed [15]. For its separation, temperature researches were carried out. EPR spectra with $g_{e f}=2.0099$ at different temperatures $(77-500 \mathrm{~K})$ are given in Fig. 5. One can see that the width and the intensity of the band vary weakly with temperature. The magnitude of an activation energy of the band, determined from a temperature dependence of a line width $\left(\Delta B=\Delta B_{\mathrm{o}} \exp \left(-E_{a} / k T\right)\right.$ [22], is equal $0.03 \mathrm{eV}$. The obtained low value of an activation energy indicates an asymmetrical paramagmetic center (PC) configuration [15]. In this case, a relaxation process in an external field does not require large energy expenses. As it is known, if the form of a EPR line depends poorly on temperature, the spin number is determined precisely enough as a square under the curve of the intensity derivative magnitude $I_{\mathrm{O}}{ }^{*}$ dependence on magnetic field induction [14]:

$S=\left(\pi / \sqrt{3} \mu_{0}\right) I_{0}^{*} \Delta B^{2}$

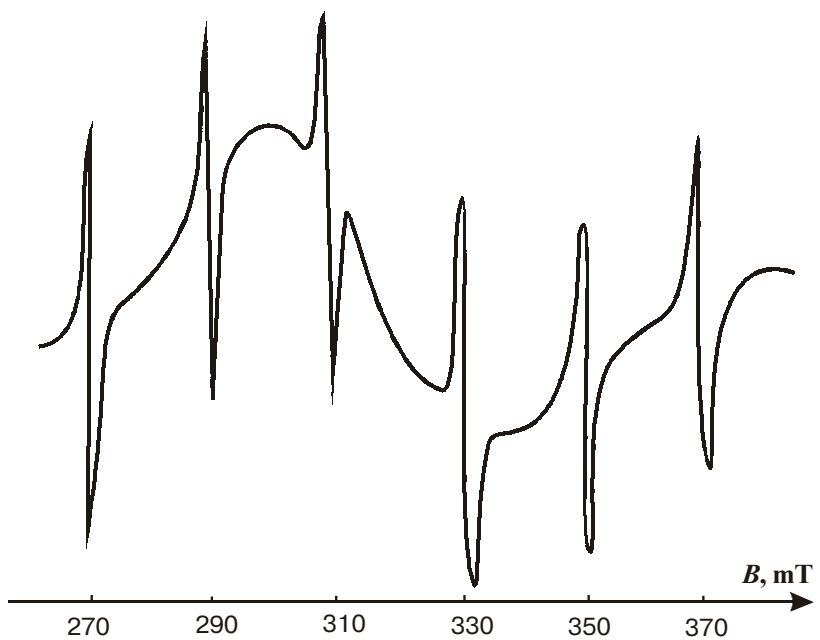

Fig. 4. EPR spectra in the $B=270-370 \mathrm{mT}$ region for $\beta-\mathrm{ZnP}_{2}: \mathrm{Mn}$ and $\mathrm{MgO}$ measured standard 
M. Kakazej et al.: Behaviour of manganese impurity in $\beta-\mathrm{ZnP}_{2}$

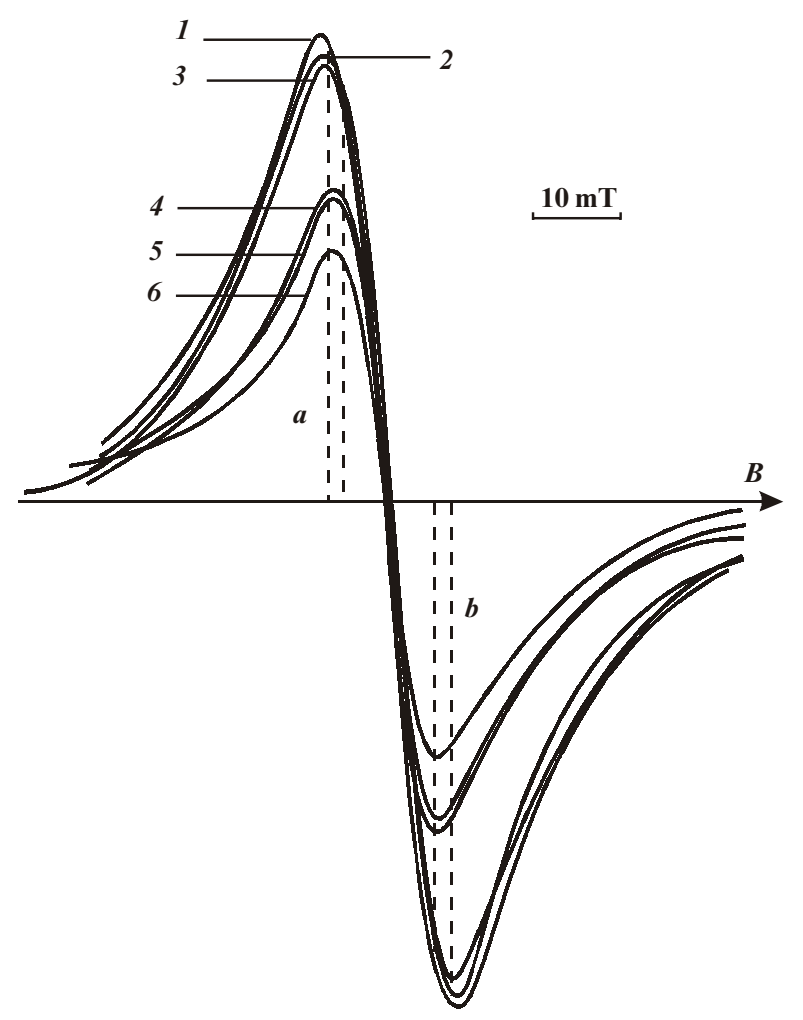

Fig. 5. EPR spectra with $g_{e f}=2.0099$ for initial $b-\mathrm{ZnP}_{2}$ : Mn at different observed temperatures: $T=77 \mathrm{~K}(I), T=200 \mathrm{~K}(2), T=500$ K (3), irradiated by electrons with the energy $E=14 \mathrm{MeV}$ (4) and annealed at $T=100{ }^{\circ} \mathrm{C}(5), T=300{ }^{\circ} \mathrm{C}(6)$. Asymmetry parameters $(a, b)$ are shown as dotted lines

Using the known value of uncoupled spins in the measured standard $(\mathrm{MgO})$ and data obtained from Fig. 4, the concentration of paramagnetic centers in zinc diphosphide responsible for a band with $g_{e f}=2.0099$ was found to be $N=1.5 \cdot 10^{17} \mathrm{~cm}^{-3}$.

In majorities of semiconductors doped by $\mathrm{Mn}$, the wide line (with a width $>10 \mathrm{mT}$ ) without HFS is ascribed to the manganese existence as unisolated centers [24, 25]. To determine a charge state of PC, to which EPR spectrum with $g_{e f}=2.0099$ responds, doped samples were annealed at three temperatures: $800^{\circ} \mathrm{C}\left(\right.$ I mode), $1000^{\circ} \mathrm{C}$ (II mode) and $1100^{\circ} \mathrm{C}$ (III mode). In the first mode the pressure of a phosphorus vapor was lower than the equilibrium one, in the second was equal to equilibrium, and in the third was higher than the equilibrium one, according to the $P-T$ - $V$ diagram for a system $\beta-\mathrm{ZnP}_{2}$ [30]. Samples were annealed in evacuated silica ampoules during 12 hours which enabled to provide equilibrium of a vapor- solid system in a layer of 300-micron width. It is known that high-temperature treatment causes appearance of imperfections, the pressure of vapor component determines the type of which in binary compounds. So, when the phosphorus vapor pressure is lower then the equilibrium one, the concentration of phosphorus vacancies - deep donors with $E_{a}=0.24 \mathrm{eV}$ (in $\beta-\mathrm{ZnP}_{2}, E_{g}=1.4$ $\mathrm{eV}$ [31]) - increases; when the pressure is more than equi- librium - the concentration of zinc vacancies, that are deep acceptors $E_{a}=0.3 \mathrm{eV}$ [32], increases. Values of concentrations of paramagnetic centers (PC) and holes after different heat treatments are given in Table 3.

Table 3. Concentrations of $\mathrm{PC}$ and charge carriers after different annealing modes

\begin{tabular}{lllll}
\hline \hline Sample & Initial & 1 mode & 2 mode & 3 mode \\
\hline $\begin{array}{l}\text { PC concentra- } \\
\text { tion, } \mathrm{cm}^{-3}\end{array}$ & $1.5 \cdot 10^{17}$ & $7 \cdot 10^{16}$ & $2 \cdot 10^{17}$ & $6 \cdot 10^{17}$ \\
\hline $\begin{array}{l}\text { Hole concentra- } \\
\text { tion, } \mathrm{cm}^{-3}\end{array}$ & $6.5 \cdot 10^{20}$ & $2 \cdot 10^{18}$ & $4 \cdot 10^{20}$ & $8 \cdot 10^{21}$ \\
\hline \hline
\end{tabular}

From the Table 3 , it is visible that the heat treatment at the first mode caused an additional compensation of PC due to appearance of donor electrons of phosphorous vacancies, and at the third mode - to $\mathrm{PC}$ recompensation. The second mode is intermediate. Such character of a modification of the PC and hole concentrations during annealing indicates the recharge of deep centers of manganese at a displacement of the Fermi level. So, the charge state of studied PC in initial crystals is close to neutral.

In order to find out its structure and position in a lattice $\beta-\mathrm{ZnP}_{2}$, let us analyze parameters of spin-Hamiltonian: $D$ (tensor of the FS), $A$ (tensor HFS) and $g$-factor.

One can see from Fig. 3 that the relative intensity of a band with $g_{e f}=2.0099$ is much higher than the intensity of a background created by non-central transitions of a fine structure (FS) in lines with others $g_{e f}$-factors. A similar background is presented in majority of $\mathrm{Mn}^{2+}$ experimental spectra with $g_{e f}=2.00$ in oxide polycrystals and glasses [33]. For the evaluation of the width of distribution of the thin structure parameter $D$ for $\mathrm{Mn}^{2+}$ in zinc diphosphide one can use the half-quantity analysis of a EPR $\mathrm{Mn}^{2+}$ structureless line with the close $g$-factor received for $\mathrm{ZnCl}_{2}$ : $\mathrm{Mn}$ [33] - the structural analog of crystals studied. The comparative analysis has shown that the parameter $\mathrm{D}$ distribution at the halfheight of a band in $\beta-\mathrm{ZnP}_{2}$ must be $\Delta D>10 \mathrm{mT}$. In binary compounds the spectrum with broad distribution of a parameter $\mathrm{D}(0.07<D<0.25)$ in Tesla can be explained by effects of composite disorder of the $\mathrm{Mn}^{2+}$ second coordination sphere and is caused by the existence of manganese ion clusters as separate phases, which are not mixed up with a basic matrix of a lattice [15]. So, the data obtained in measurements of the Mössbauer effect [34] and EXAFS [35] in polycrystals showed that ions with the $d^{5}$ configuration in interstate positions cause a broad background absorption.

Comparing EPR spectra in Fig. $3(a)$ and $(b)$ one can see that in the magnetic field region of $B=290-370 \mathrm{mT}$, where there is a broad band in highly doped samples, in crystals of $\beta-\mathrm{ZnP}_{2}$ with small $\mathrm{Mn}(0.01 \%)$ content there are singularities with $g_{e f}=2.17 \mathrm{i} g_{e f}=1.92$ and with an identical parameter $A=6.2 \mathrm{mT}$. From the EPR spectrum modeling in polycrystals [16] it is clear that the presence of separate singularities responds to the existence of isolated $\mathrm{Mn}^{2+}$ in a lattice. For $\mathrm{Mn}^{2+}$ ions an additional information about 


\section{Kakazej et al.: Behaviour of manganese impurity in $\beta-\mathrm{ZnP}_{2}$}

possible number of the close neighbours can be received by the HFS constant $(A)$ that determines PC coordination and rises, when the coordination number increases [15]. PC with $g_{e f}=2.17 \mathrm{i} g_{e f}=1.92$ have the greatest value of the $A$ parameter, therefore, their first coordination sphere should have the greatest amount of ligands, comparing with other centers in zinc diphosphide. Such position in a lattice can belong to $\mathrm{Mn}_{\mathrm{i}}$ intersticial.

Obviously, two different structural positions of manganese in a lattice with the identical coordination of centers in a weak ligand field respond to ascertained singularities. Then, the formation of a broad band with $g_{e f}=2.0099$, when the manganese concentration increases, can be caused by two factors - displacement of the $g$-factor of singularities with $g_{e f}=2.17$ and 1.92 as well as the extension of FS lines. It is known [14] that the displacement of the magnitude of the $g$-factor is a result of modification of spin-orbit bonds in $\mathrm{PC}$, arising in the course of restructurization, and lowering of a local PC symmetry causes extension of FS lines. Such reorganization can be performed by defect clusters that have an asymmetrical configuration, as it was stated above. It is also necessary to mention that the formation of intersticial clusters is peculiar to atoms that diffuse easily [36].

So, studied properties of spin-Hamiltonian parameters of a band with $g_{e f}=2.0099$ indicate that the band is caused by clusters of manganese atoms, which are embedded impurities in $\beta-\mathrm{ZnP}_{2}$.

\section{$E P R$ spectra of manganese ions in the region $B=150-180 \mathrm{mT}$}

EPR spectrum in the region $B=150-180 \mathrm{mT}$ (Fig. 6 ) is characteristic to manganese ions in an axial crystalline field [15] and is satisfactory described by spin-Hamiltonian :

$\Re=g \beta(H S)+D\left[S_{z}^{2}-1 / 3\{S(S+1)\}\right]+A(S I)$

The magnitude $D$ can be evaluated by measuring the relative intensity I of hyperfine forbidden transitions (HFT) $-\{-1 / 2, m \leftrightarrow+1 / 2, m= \pm 1\}$. For polycrystals the intensity can be expressed by the spin-Hamiltonian constants [37]:

$$
I_{\mathrm{HTF}}=\left(512 D^{2} / 15 H_{\mathrm{o}}{ }^{2}\right)(35 / 4-m(m+1))
$$

Ratio of amplitudes of low field HTF transition $(-1 / 2$, $-5 / 2 \leftrightarrow+1 / 2,-3 / 2)$ to appropriate hyperfine CT components $(-1 / 2,-5 / 2 \leftrightarrow+1 / 2,-5 / 2)($ at $A<0)$ is equal 0.10 and, accordingly, $D=7 \mathrm{mT}$. For the more precise definition of spin-Hamiltonian constants let us use the expression for an angular dependence of a spectral position of thin structure lines [37]:

$$
\begin{aligned}
& H=H_{\mathrm{o}}-D\left(M_{S}-1 / 2\right)\left(3 \cos ^{2} \Theta-1\right)+\left(D^{2} \cos ^{2} \Theta \times\right. \\
& \left.\times \sin ^{2} \Theta / 2 H_{\mathrm{o}}\right)\left[4 S(S+1)-24 M_{S}\left(M_{S}+1\right)-9\right]-\left(D^{2} \times\right. \\
& \left.\times \sin ^{4} \Theta / 8 H_{\mathrm{o}}\right)\left[2 S(S+1)-6 M_{S}\left(M_{S}-1\right)-3\right]-A m-\left(A^{2} / 2 H_{\mathrm{o}}\right) \times
\end{aligned}
$$

$\times\left[I(I+1)-m^{2}+\left(2 M_{S}-1\right) m\right]-\left(D^{2} A \sin ^{2} \Theta \cos ^{2} \Theta / 2 H_{\mathrm{o}}{ }^{2}\right)\left\{\left[\left(M_{S}{ }^{2}-\right.\right.\right.$ $\left.-S(S+1))^{2}-M_{S}^{2}\right] / M_{S}-\left[\left(M_{S}-1\right)^{2}-S(S+1)^{2}-\left(M_{s}-\right.\right.$ $\left.\left.1)^{2}\right] /\left(M_{s}-1\right)\right\} m-\left(D^{2} \sin ^{4} \Theta / 8 H_{\mathrm{o}}\right)\left\{\left(M_{s}\left[2 M_{S}^{2}+1-2 S(S+\right.\right.\right.$ 1) $\left.-\left(M_{s}--1\right)\left[2\left(M_{s}-1\right)^{2}+1-2 S(S+1)\right]\right\} m$

In polycrystals, the $\mathrm{PC}$ number of the spectrum in an interval of fields $H \mathrm{i} H+\Delta H$, is proportional to magnitude $\Delta n$, depending on signal intensity $I(\Theta)$ by the formula:

$\Delta n=\sin \Theta I(\Theta) \Delta H /(d H / d \Theta)$,

where $\Theta$ is an angle between a crystalline axes and magnetic field. The maximum of the intensity in a background EPR signal occurs under condition $(\Delta n / \Delta H) \rightarrow \infty$. By the differentiating the expression (5) on an angle and putting an obtained value $(d H / d \Theta)$ into the equation (6), one can receive values of angles of the particle orientation, at which there should be maximal EPR signal in an absorption curve for different transitions:

for $(-1 / 2, m) \leftrightarrow(+1 / 2, m)$ - angles $\Theta_{1}=90^{\circ}, \Theta_{2}=$ $=\operatorname{arctg}\{(4-36 \mathrm{Am} / \mathrm{H}) /(5-37 \mathrm{Am} / \mathrm{H})\}^{-1 / 2}$

for $( \pm 1 / 2, m) \leftrightarrow( \pm 3 / 2, m)$ and $( \pm 3 / 2, m) \leftrightarrow( \pm 5 / 2, m)-$ angle $\Theta=90^{\circ}$.

So, using calculated values $\Theta_{1}$ and $\Theta_{2}$ for transitions $(-1 / 2, m) \leftrightarrow(+1 / 2, m)$ and experimentally obtained magnitudes of the strength of a magnetic field, which respond to maximum of signals - responses of FS transitions (Fig. 6), using the formula (5), the interval of modifying the $A$ parameter was defined as $A=(6.4 \pm 0.1) \mathrm{mT}$.

In low field $(a-e)$ and high field $\left(a^{\prime}-e^{\prime}\right)$ parts of the spectrum (Fig. 6) observed are weak lines caused by signalsresponses of FS transitions: $( \pm 1 / 2, \pm 5 / 2) \leftrightarrow( \pm 3 / 2, \pm 5 / 2)$ and $( \pm 3 / 2, \pm 5 / 2) \leftrightarrow( \pm 5 / 2, \pm 5 / 2)$. By determining their positions concerning the nearest central transitions and by comparing them with the values eveluted by the formula (5), there were found the interval of a modification of a parameter $D=6.5 \pm 0.2 \mathrm{mT}$ and (using the equation of the work [15]) - parameter $E=0.5 \pm 0.1$.

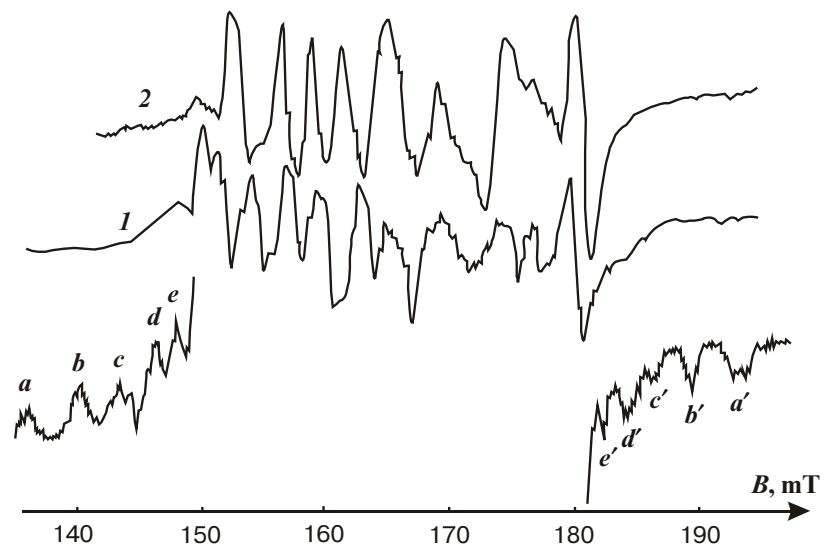

Fig. 6. EPR spectra with $g_{e f}=4.3$ for initial (1) and irradiated (2) $b$ $\mathrm{ZnP}_{2}$ : Mn crystals 


\section{Kakazej et al.: Behaviour of manganese impurity in $\beta-\mathrm{ZnP}_{2}$}

Thus, relation between obtained spin-Hamiltonian constants $D$ and $E(D / E \neq 3)$, the magnitude of $g$-factor $\left(g_{\text {ef }}=30 / 7 \approx 4.3\right.$ ), and also dependence of the absorption intensity of a EPR signal on the manganese concentration (Fig. 3, $a$ and $b$ ) indicate its connection with $\mathrm{Mn}^{2+}$ ions in strong crystalline field of four -multiple coordination with weak rhombic distortion [15]. The computer study of the Roentgen-structural analysis data of $\beta-\mathrm{ZnP}_{2}$ crystals (space group $\left.\mathrm{P} 2{ }_{1} / c=\mathrm{C}_{2 \mathrm{~h}}{ }^{5}\right)$ (Table 4 ) has shown that all structural positions of zinc atoms are tetrahedrally coordinated. So, the ions of manganese, replacing zinc atoms in spots of a crystalline lattice are responsible for the EPR spectrum with $g_{e f}=4.3$.

As it is clear from the Table 4, the zinc atom has two nonequivalent positions in the first coordination sphere: the atom of zinc is surrounded by four atoms of phosphorous - the $1^{\text {st }}$ state, and, if atom of zinc is surrounded by two atoms of zinc and two atoms of phosphorous, - the $2^{\text {nd }}$ state. In opposition to the tetrahedral $\alpha-\mathrm{ZnP}_{2}$ modification there are bonds metal-metal $\mathrm{Zn} 2-\mathrm{Zn} 2$, available in $\beta-\mathrm{ZnP}_{2}$. So, when zinc atoms are substituted by manganese atoms, three possible tetrahedrons can be received in the structure $\beta-\mathrm{ZnP}{ }_{2}$, which differ by the first coordination sphere of an Mn ion environment 1) Mn (Zn1) and four P; 2) Mn (Zn2), Zn2 and three P; 3) Mn (Zn1), Mn (Zn2) and two P. For each tetrahedron it is possible to count an ion degree of four bonds as a sum of electroconductivity differences of bound atoms. The calculation shows, that there is the greatest ion degree of bonds for the tetrahedron of the first type, at which manganese ion substitutes the zinc atom in a state $\mathrm{Zn}_{1}$; and the least ionization degree is for the third type of tetrahedron, in which two manganese atoms substitute the two zinc atoms in a state $\mathrm{Zn} 2$.

Calculations of EPR spectra in all researched region have shown that singularities with $g_{e f}=4.3$, have the greatest value of an HFS parameter $(A=6.4 \mathrm{mT})$. It is known [15] that the magnitude of a parameter $A$ for $\mathrm{Mn}^{2+}$ increases with a diminution of a bond covalent degree and magnification of their length. That is, the tetrahedron with the greatest ion degree in binary composition responds to the singularity with the greatest parameter $A$. By comparing values of bond ion degrees in tetrahedrons mentioned and the distance between ligand in them (Table 4), it is possible to state, that PC of tetrahedral kind, in which $\mathrm{Mn}^{2+}$ substitutes atom of zinc in a $\mathrm{Zn} 1$ position, respond to the singularity with $g_{e f}=4.3$.

The presence of singularities with $g_{e f}=2.55, g_{e f}=4.3$ and $g_{e f}=20$ in highly doped crystals indicates the existence of three types of centers in a strong ligand field; $\mathrm{Mn}^{2+}$ has a different environment in these centers. One can explain it as the presence of structural nonequivalent positions of atoms in a lattice of $\beta-\mathrm{ZnP}_{2}$ crystals. The existence of singularities with $g_{e f}>20$ in an EPR spectrum (Fig. 3) indicates a high degree of continual violations of the near order in highly doped crystals.

Thus, the manganese atoms can occupy a series of structural - nonequivalent positions in a zinc diphosphide lattice, forming some types of paramagnetic centers (penetration and substitution), which can be indicators of a local electrical field at defect creation processes .
Table 4. Interatomic distances and angles (according to the direction of atom bond with first ligand) in black $\beta-\mathrm{ZnP}_{2}$.

\begin{tabular}{|c|c|c|c|}
\hline Atom & Ligands & Distances, $\AA$ & Angle, ${ }^{\circ}$ \\
\hline \multirow[t]{4}{*}{$\mathrm{Zn1}$} & $\mathrm{P}_{2}$ & 2.306 & - \\
\hline & $\mathrm{P}_{3}$ & 2.424 & 110.2 \\
\hline & $\mathrm{P}_{4}$ & 2.335 & 108.2 \\
\hline & $\mathrm{P}_{4}$ & 2.341 & 113.2 \\
\hline \multirow[t]{4}{*}{$\mathrm{Zn} 2$} & $\mathrm{Zn} 2$ & 2.433 & - \\
\hline & $\mathrm{P}_{1}$ & 2.343 & 119.5 \\
\hline & $\mathrm{P}_{2}$ & 2.375 & 109.6 \\
\hline & $\mathrm{P}_{3}$ & 2.324 & 108.5 \\
\hline \multirow[t]{4}{*}{$\mathrm{P}_{1}$} & $\mathrm{Zn} 2$ & 2.343 & - \\
\hline & $\mathrm{P}_{1}$ & 2.255 & 128.3 \\
\hline & $\mathrm{P}_{2}$ & 2.388 & 101.2 \\
\hline & $\mathrm{P}_{3}$ & 2.332 & 102.0 \\
\hline \multirow[t]{4}{*}{$\mathrm{P}_{2}$} & $\mathrm{Zn1}$ & 2.306 & - \\
\hline & $\mathrm{Zn} 2$ & 2.375 & 118.0 \\
\hline & $P_{1}$ & 2.388 & 122.8 \\
\hline & $\mathrm{P}_{4}$ & 2.221 & 107.5 \\
\hline \multirow[t]{4}{*}{$\mathrm{P}_{3}$} & $\mathrm{Zn1}$ & 2.424 & - \\
\hline & $\mathrm{Zn} 2$ & 2.324 & 118.9 \\
\hline & $\mathrm{P}_{1}$ & 2.332 & 120.7 \\
\hline & $\mathrm{P}_{4}$ & 2.168 & 104.6 \\
\hline \multirow[t]{4}{*}{$\mathrm{P}_{4}$} & $\mathrm{Zn1}$ & 2.335 & - \\
\hline & $\mathrm{Zn} 1$ & 2.341 & 107.9 \\
\hline & $\mathrm{P}_{2}$ & 2.221 & 110.1 \\
\hline & $\mathrm{P}_{3}$ & 2.168 & 113.5 \\
\hline
\end{tabular}

\section{EPR of manganese ions in irradiated zinc diphosphide crystals}

In Fig. 5 EPR spectra of ions of manganese with $g_{e f}=2.0099$ are shown after irradiation by electrons with $E=14 \mathrm{MeV}$ and annealing at temperatures, which respond to the stage ends of isochronous electroconductivity annealing [7]. One can see that the magnitude of amplitude of the first derivative and a line width $\Delta \mathrm{B}$ are decreased on irradiation, which indicates a falling of PC concentration (equation 2). Besides, the amplitude of a high-field part of the band $(b)$ is $10 \%$ larger then the low-field part $(a)$. After irradiation the parameter of asymmetry $(a / b)$ is nearly equal to one. Such character of a band asymmetry change indicates a lowering of spin-orbit bonds of initial PC after irradiation [14]. It is obviously that $\mathrm{Mn}_{\mathrm{i}} \mathrm{PC}$ intersticial clusters are rebuilt due to the interaction with radiation defects. The character of change can be judged by estimating average distances between $\mathrm{PC}$ as the band spin-packet width $\Delta \mathrm{B}$ using Eq. (3), in the case of the dipole-dipole interaction between PC parts [38]: 
M. Kakazej et al.: Behaviour of manganese impurity in $\beta-\mathrm{ZnP}_{2}$

$r \approx\left(a \Delta B / c \mu_{\mathrm{o}}\right)^{-1 / 3}$

Factors $a$ and $c$ in the equation (3) are special compound constants. The ratio of average distances $r_{\mathrm{o}} / r_{f} \approx 50$ one can receive by measuring the spin-packet width of the band studied in initial $\left(\Delta B_{\mathrm{o}}\right)$ and irradiated $\left(\Delta B_{f}\right)$ crystals (Fig. 5). That is, distances between PC increase after irradiation.

To understand the nature of radiation defects, which influence on EPR spectrum parameters of irradiated samples, let us analyze the spectrum in the interval $B=250-277 \mathrm{mT}$. The $\mathrm{Mn}^{2+} \mathrm{EPR}$ spectra in this interval for initial and irradiated crystals are shown in Fig. 7. It is obvious that the introduction of radiation defects reduces singularity intersities and the magnitude of the $g$-factor. The displacement of the $g$ factor of the spectrum is observed in a case of continual violations of long-distance sphere of PC [17]. The EPR line inhomogeneous extension of impurity PC, observed as the change of ratioes of amplitudes of allowed and forbidden FS transitions (in particular, in the high - field part) (Fig. 7), has shown the presence of fields of electrically active imperfections in irradiated samples [37]. Such imperfections in zinc diphosphide are complexes of vacancies of zinc and phosphorous in different coordinate positions of phosphorous sublattice, which are main conductivity compensators in irradiated crystals [7].

Modifications of $A$ parameter and PC concentration of two singularities with $g_{e f}=4.3$ and $g_{e f}=2.0099$ after irradiation and annealing are shown in Fig. 8. One can see from this figure that the PC concentration with $g_{e f}=4.3$ increases and with $g_{e f}=2.0099$ decreases. The opposite radiation influence on the concentration of these centers confirms a different nature of centers. An isochronous annealing has shown (Fig. 8) that at $T=300^{\circ} \mathrm{C}$, when the centers are disintegrated on separate mobile vacancies [18], the PC concentration with $g_{e f}=2.0099$ continues to drop, and $g_{e f}=4.3$ - to increase. Thus, changes observed in EPR spectra of irradiated crystals are connected with the $\mathrm{Mn}_{\mathrm{i}}$ capture by radiation induced vacancies and the transition of atoms of manganese from the state of penetration impurity to the substitution state.
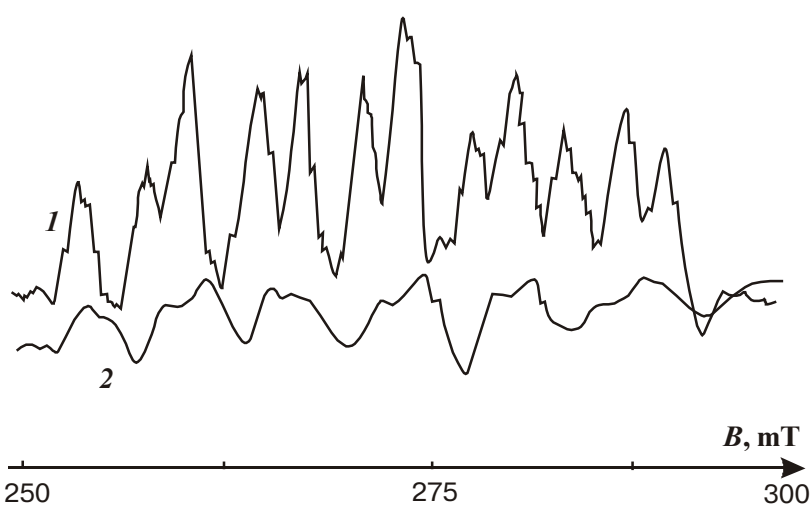

Fig. 7. Manganese ion EPR spectra in the $B=250-300 \mathrm{mT}$ region for initial (1) and irradiated (2) $\beta-\mathrm{ZnP}_{2}$ : Mn crystals

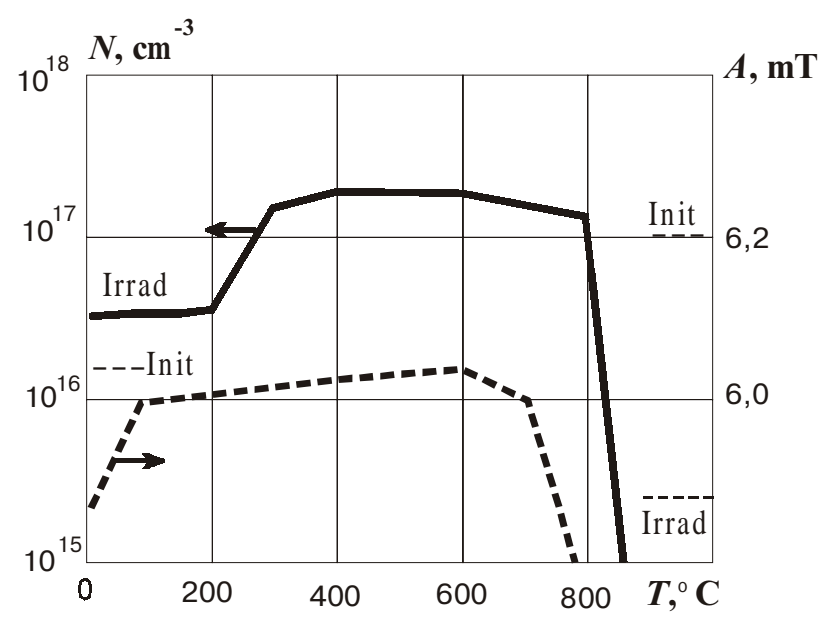

a)

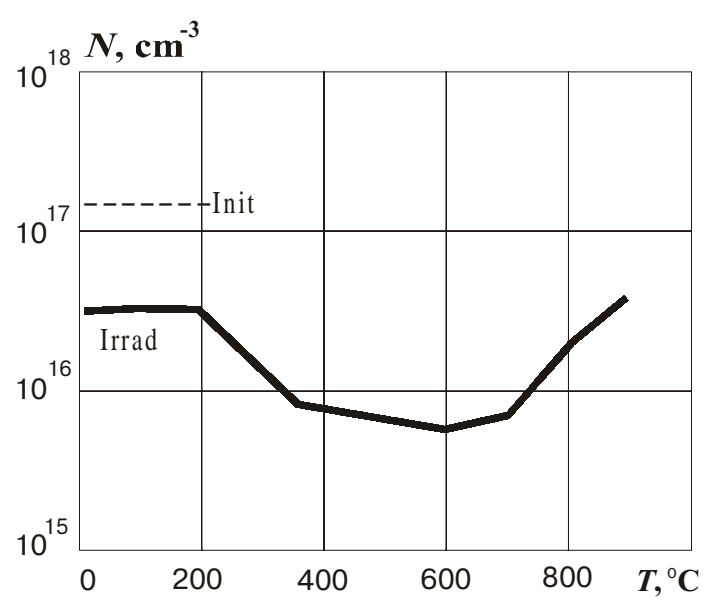

b)

Fig. 8. Changes of $\boldsymbol{A}$ parameter of EPR and PC concentrations with $g_{e f}=4.3(A)$ and $g_{e f}=2.0099(B)$ for irradiated and isochronically annealed crystals

One can see from Fig. 6 that the low - field group of spectrum peaks with $g_{e f}=4.3(a, b)$ is displaced into the large field region, thus the position of high-field singularities $(c, d)$ remains constant. The $A$ parameter decreases as well. The anomaly of local widths of $\mathrm{Mn}^{2+}$ singular lines in binary compositions one can explain (in the framework of one-harmonic model) as the amplitude fluctuations of lattice atom displacements [39]. Besides the lack of the splitting of resonance lines can testify the preservation of a PC local symmetry [40]. The parameter $A$ diminution after irradiation is, as it has been already mentioned, the direct evidence of the increase of the covalent degree in the first coordination sphere in the environment of manganese, which substitute zinc in the position $\mathrm{Zn} 1$. All this indicates the rebuilding of the PC structure with $g_{e f}=4.3$ after irradiation with involvement of imperfections of a substitution type.

An isochronous annealing has shown (Fig. 8) that the parameter $A$ is almost completely restored at temperatures considerably lower then annealing temperature of vacancy complexes - main electrically active radiation defects of 


\section{Kakazej et al.: Behaviour of manganese impurity in $\beta-\mathrm{ZnP}_{2}$}

zinc diphosphide crystals [18]. That is, EPR spectrum study has shown that at $T=80^{\circ} \mathrm{C}$ radiation defects are annealed, which do not influence on electroconductivity [18] but act in exciton IR reflection spectra [41]. Obviously, these defects are simpler than vacancy complexes. Taking into account the high mobility of primary defects in zinc diphosphide at $T<300 \mathrm{~K}$ [42], antistructural imperfections could be such substitute defects. Our proposition is confirmed by the calculation of bond ion degrees. The replacement by zinc atom of one phosphorous atom from the manganese environment, to which the spectrum with $g_{e f}=4.3$ (tetrahedron of a first type) responds, reduces a bond ion degree from the value 1.84 up to 1.78 . Thus, the $A$ parameter changes in this temperature interval are caused by antistructural imperfections of a type $\mathrm{Zn}_{\mathrm{P}}$ appearing in irradiated zinc diphosphide crystals.

Beginning from temperature $T=600^{\circ} \mathrm{C}$ the $A$ parameter of the spectrum with $g_{e f}=4.3$ begins to drop, and at $T>850^{\circ} \mathrm{C}$ the EPR spectrum in this g-factor region is absent. As one can see from Fig. 8, at these temperatures the concentration of singularities with $g_{e f}=2.0099$ increases. That is, ions of manganese in the temperature interval of $850-900^{\circ} \mathrm{C}$, diffusing from crystalline lattice spots, create interstate PC complexes. Such character of the PS rebuilding reduces the thermohardness of crystall electrical parameters (Table 1).

\section{Conclusions}

Thus, from results received by EPR, Hall effect, optical and Roentgen-structural analysis of black zinc diphoshide crystals doped by manganese it is possible to conclude:

1. The existence of singularities with $g_{e f}=20, g_{e f}=4.3$, $g_{e f}=2.55$ and $g_{e f}=2.0099$ in the EPR spectrum are connected with spatially - nonequivalent positions of manganese atoms in a crystalline lattice of $\beta-\mathrm{ZnP}_{2}$.

2. The broad unstructured band with $g_{e f}=2.0099$ and the width $\Delta B=14 \mathrm{mT}$ in initial crystals is caused by of manganese interstate clusters (penetration impurity) with charge state close to neutral.

3. EPR spectra with $g_{e f}=4.3$ belongs to $\mathrm{Mn}^{2+}$ ions, which substitute atoms of zinc in the state $\mathrm{Zn} 1$.

4. Intersticial atoms of manganese in crystals irradiated by electrons with $E=14 \mathrm{MeV}$ while interacting with of vacancies of zinc have become impurities of substitution.

5. $\mathrm{Mn}(\mathrm{Zn} 1)$ centers of substitution cause high thermal stability of electrical characteristics of $\beta-\mathrm{ZnP}_{2}: \mathrm{Mn}$ crystals.

6. The antistructural defects of a $\mathrm{Zn}_{P}$ type arising after irradiation by electrons with $E=14 \mathrm{MeV}$, can be annealed at temperature $T=80^{\circ} \mathrm{C}$

\section{Abbreviations}

$C T$ - central transitions

$H F S$ - hyperfine forbidden transitions

$P C$ - paramagnetic centers

$F S$ - fine structure

$H F S$ - hyperfine structure

\section{References}

1. N. N. Syrbu. Optoelectronic properties of $A^{I I} B^{V}$ group compounds, Shtiica, Kishinev (1983) (in Russian ).

2. Gintautas-Jurgis Babonais. An optical anisotropy of wide-zone semiconductors. Dr. Sci. Theses, Vilnius (1990) (in Russian).

3. V. A. Zujev, V. G. Popov, B. N. Romaniuk. A condenser photoe.m.f. in ion bombing crystals $\mathrm{ZnP}_{2}$ and $\mathrm{CdP}_{2}$ // Surface: physics, chemistry, mechanics. 7, pp. 94-97 (1992) (in Russian).

4. Sigusaki Mitsuri, Arimito Osamu. Intersystem conversion between singlet and triplet exiting states in $\beta-\mathrm{ZnP}_{2} / /$ Repts. Res. Lab. Surface Sci. / Fac. Okayama Univ. 7(5), pp. 207-213(1995).

5. I. S. Gorban', M. M. Bilyj. The phase diagram of dielectric biexciton fluid in $\beta-\mathrm{ZnP}_{2}$ crystals // Ukr. Phys. Journ. 41 (9), pp.840-846 (1996) (in Ukrainian).

6. S. Garasevich, P. Koreniuk, O. Slobodianiuk at.al. Isotope effects in spectra of light combination scattering of gyrotropic crystals of zinc and cadmium diphosphides// Magazine Phis. Researches, 4(2), pp.202-207(2000) (in Ukrainian).

7. A. P. Kudin. An action of particle flows of high energies on thin defect structure of zinc diphosphide monocrystals//Physics and chemistry of material treating, 1, pp. 33-37 (2000) (in Russian).

8. N. P. Iljin, A. E. Vasiljev, V. F. Masterov. A basic state of the iron group transitional elements in gallium arsenide and phosphide // Phys. Tech. Polupr. 26 (11), pp.1878-1883 (1992) (in Russian).

9. V.V. Novikov. Ionic conductivity of $\mathrm{ZnP}_{2}$ and $\mathrm{CdP}_{2}$ crystals // Reports of AN BSSR, 26, p.697(1982) (in Russian).

10. N.S. Korets, I.I. Tychyna, V.B. Chornogorenko. Doping of phosphide semiconductor monocrystals by phosphide of transitional metals // Inorganic materials 33(12), pp.1432-1434 (1997).

11. G.A. Balchajtis, N. S. Korets, Z. V. Janushkjavichjus. Doping of cadmium diphosphide crystals // Izv. AN SSSR, Inorganic materials, 22(4), pp. 536-539(1986) (in Russian).

12. A.P. Kudin. A special laboratory practical work from physics of solids. September, Kiev, p.117 (1997) (in Ukrainian).

13. P.Ju. Zilinskas, A.P. Sakalas. A measurement of a Hall effect in high-resistance samples // Polupr. Techn. and Electr., 3, pp. 238-241 (1972) (in Russian).

14. M.V. Vlasova, N.G. Kakazej. An electronic paramagnetic resonance in mechanically desroyed solids. Naukova dumka, Kyiv, p.196 (1979)

15. Ja.G. Kliava. EPR spectroscopy of unordered solids. Zinatne, Riga, p. .231 (1988) (in Russian).

16. S.K Misra. Parameter $\mathrm{Mn}^{2+}$ of electron paramagnetic resonance // Physica (B) 203(1-2), pp. 193-200(1994).

17. Vlasova, N. G. Kakazej. A structure of a powder of silicon after plasma-chemical treating // Inorganic mat. 35 (5), pp.519-523 (1997) (in Russian).

18. V. P. Tartachnyk. Radiation defects in semiconducting $A^{3} B^{5} i$ $A^{2} B^{5}$ phosphides. Dr. Sci. Theses, Chernivtsi, p. 15(1993) (in Ukrainian).

19. P.K. Khowask. Theoretical study of $\mathrm{A}^{3} \mathrm{~B}^{5}$ defects influence // Phys. Rev. (B), 49(16), pp. 10991-10997 (1994).

20. G. K. Ipolitova, M. N. Omelianovsky. A behaviour of Fe impurity in InP and covalence influence on a EPR spectrum of an $\mathrm{Fe}^{3+}$ ion in the compounds of $T_{d}$-symmetry // Phys. Tech. Polupr., 11(7), pp. 654-660(1977) (in Russian).

21. P. G. Baranov. The radiospectroscopy of wide-zone semiconductors Si and GaN // Phys. Tech. Polupr., 41(5), pp.789793 (1999) (in Russian).

22. I. A. Gejfman, I. S. Golovina, I. V. Kozlov. EPR and dielectric relaxation of $\mathrm{Fe}^{3+}$ in $\mathrm{KTaO}_{3}$ // Sol. St. Phys., 11(7), pp. 861864 (1997) (in Russian).

23. F.K. Koschnict, M. Rac. Anomalous spin-lattice relaxation in InP: $\mathrm{Fe}^{3+}$ and $\mathrm{BaFBr}: \mathrm{Eu}^{2+}$ examined of electron paramagnetic resonance // J. Phys. Condens. Matter. 5, pp. 733-746 (1993).

24. M. Palczewska. Characterisiria ESR paramagnetic defects of $\mathrm{A}^{3} \mathrm{~B}^{5}$ // Praze ITME 45, p.1-54 (1995). 


\section{Kakazej et al.: Behaviour of manganese impurity in $\beta-\mathrm{ZnP}_{2}$}

25. M.L. Mejl'man, M.I. Samojlovich. Introduction into EPR spectroscopy of activated crystals. Atomizdat, M., p.272 (1977) (in Russian).

26. E. Brailovski, I. Megela, N. Pambuchchyan, V. Teslenko. ESK Study of electron-irradiated InP:Fe // Phys. Stat. Sol. (a) $\mathbf{7 2}$, K109-111 (1982).

27. M. Rakowsky, W. Kuhn, W. Lauderdale. Electron paramagnetic resonance study of nature acceptor in as-grown $\mathrm{ZnGe} \mathrm{P}_{2} / / \mathrm{Appl}$. Phys. Lett. 13 (13), pp.1615-1617 (1994).

28. M. Giles, F. Halliburton. ESR of $\mathrm{ZnGe}_{2} / /$ Appl. Phys. Lett. 66(14), pp.1758-1762 (1995)

29. P. Zapol. Atomic defects of ZnGe $\mathrm{P}_{2} / /$ J. Appl. Phys., 79(2), pp. 671-675 (1996)

30. N.Ja. Jakimovich, V.A. Rubcov, V.M. Truchan. A phase equilibrium in a system Zn-P-As-Cd // Inorganic materials, 32 (7), pp. 799-803 (1996) (in Russian).

31. V.B. Lasarev, V.Ja. Shevchenko, Ja.H. Grinberg, V.V. Sobolev. Semiconducting junctions of $A^{2} B^{5}$ group. Nauka, M., p.213 (1978) (in Russian).

32. I. Gorban, G. Grischchenko, A. Sakalas. Electrical properties of $\mathrm{ZnP}_{2}$ single crystals of tetragonal and monoclinic modifications // Phys. Stat. Sol. 48, pp.329-340 (1978).

33. A. Chatelain, R. Weeks. Devitrification ordering and vitrification disordering processes: ESR study of $\mathrm{ZnCl}_{2}: \mathrm{Mn}^{2+} / /$ J.Chem. Phys. 52, pp.3758-3764 (1970).

34. C. Sunandana, P. Jagannathan. ESR and Mцssbauer studies of $\mathrm{Fe}^{3+}$ ion in calcium boro-aluminade glasses. // Solid. St. Comm. 53, p.985 (1985).
35. G. Licheri, G. Paschina. On the coordination of iron ions in sodium borosilicate glasses. III. An EXAFT investigation // $J$. Now-crystal. Solids. 72, p.211 (1985).

36. G. E. Arkhangelskij, E. E. Bukke, T. E. Voznesenskaja. A character of introduction and distribution of an impurity in crystals $\mathrm{ZnSe}$, grown from their steam phases. In book "The synthesis and the growth of perfect crystals and semiconductingfilms". Nauka, Novosibirsk, p.155 (1981) (in Russian).

37. M. V. Vlasova, S. I. Gorbachuk, N. G. Kakazej, V. M. Mel'nik. $\mathrm{Mn}^{2+} \mathrm{EPR}$ in a lithographic stone deformed at high pressure // $Z P S$, 1, pp.77-82 (1984) (in Russian).

38. I. M. Zaritcky. EPR, DENR and spin relaxation in semiconducting polycrystals // Sol. St. Phys., 38 (2), p.419-425 (1996) (in Russian).

39. M. P. Trubicyn. Fluctuation wideness of $\mathrm{Mn}^{2+}$ lines in a disproportionate phase of crystals $\mathrm{Rd}_{2} \mathrm{ZnCl}_{4}$ // Sol. St. Phys., 41 (9), p.1668 (1999) (in Russian).

40. M. P. Trubicyn, V. D. Volansky. Study by EPR method of local fluctuations near segnetoelectric phase transition in $\mathrm{Li}_{2} \mathrm{Ge}_{7} \mathrm{O}_{15}$ : $\mathrm{Mn}^{2+}$ crystals // Sol. St. Phys., 40(1), p.111 (1998) (in Russian).

41. A.P.Kudin. Radiation-induced changes in infrared spectrum of black zinc diphosphide monocrystals // Semiconductor Phys. Quantum Electronics and Optoelectronics. 3(2), pp.161-164 (2000).

42. Z.Januchkevichus, V. Koval, A. Kudin, A. Sodeika. Radiation defects in single crystals of zine diphosphide // Phys. Stat. Sol. (a) 81. pp. K191-193 (1984). 